\title{
Estudio descriptivo de la capacidad de emprendimiento de los alumnos del Instituto Tecnológico de Sonora
}

\section{Descriptive study of the entrepreneurship capacity of the students of the Instituto Tecnológico de Sonora}

\author{
BUELNA-PEÑUÑURI, Ramiro Arnoldo†, LÓPEZ-PARRA, María Elvira* y ORTEGA-ARREOLA, \\ Jorge
}

Instituto Tecnológico de Sonora. Calle 5 de Febrero 818, Centro, Urb. No. 1, 85000 Cd Obregón, Son.

ID $1^{\mathrm{er}}$ Autor: Ramiro Arnoldo, Buelna-Peñuñuri / ORC ID: 0000-0001-7364-9159, CVU CONACYT ID: 290441

ID $1{ }^{\text {er }}$ Coautor: María Elvira, López-Parra / ORC ID: 0000-0003-3552-3517, Researcher ID Thomson: S-5729-2018, CVU CONACYT ID: 252600

ID $2^{\text {do }}$ Coautor: Jorge, Ortega-Arreola / ORC ID: 0000-0003-3205-396X

DOI: $10.35429 / J E T .2019 .10 .3 .1 .8$

Recibido: 10 de Octubre, 2019; Aceptado 28 de Diciembre, 2019

\section{Resumen}

El Instituto Tecnológico de Sonora (ITSON) es una universidad preocupada por el desarrollo integral de sus estudiantes, desarrollando la capacidad de emprendimiento a través de sus dimensiones. El objetivo de esta investigación fue identificar la capacidad de emprendimiento de los estudiantes a través de un estudio descriptivo y diferencia de medias, que permita el conocimiento de los niveles de percepción en sus dimensiones. Los participantes fueron estudiantes inscritos en emprendimiento y se aplicó el instrumento a una muestra de 40 estudiantes. El tipo de investigación fue descriptiva con diferencia de medias de cada género. El instrumento se adaptó de Mavila, Tinoco y Campos (2009) con una escala de Likert que va del 5 al 1 donde 5 es nunca y el 1 siempre. El Alfa de Cronbach fue de 0.908. En cuanto a los hallazgos, en las dimensiones se observó que la percepción más alta con una media de 1.5000 corresponde a realización personal y la más baja con 1.9958 a relacionarse socialmente, ambas en el rango de aceptable. En cuanto a la diferencia de medias sobre el género, se observó que no existe diferencia significativa. Se concluye que los estudiantes del ITSON perciben que tienen una capacidad de emprendimiento.

Capacidad de emprendimiento, Emprendimiento, Dimensiones del emprendimiento

\begin{abstract}
The Instituto Tecnologico de Sonora (ITSON) is a university concerned about the integral development of its students, developing the capacity of entrepreneurship through its dimensions. The objective of this research was to identify the entrepreneurship capacity of the students through a descriptive study and difference of means, which allows the knowledge of the levels of perception in their dimensions. The participants were students enrolled in entrepreneurship and the instrument was applied to a sample of 40 students. The type of research was descriptive with the difference of means of each gender. The instrument was adapted from Mavila, Tinoco \& Campos (2009) with a Likert scale that goes from 5 to 1 where 5 is never and 1 always. Cronbach's alpha was 0.908. Regarding the findings, in the dimensions it was observed that the highest perception with an average of 1.5000 corresponds to self-realization and the lowest one with 1.9958 to social relation, both in the acceptable range. Regarding the difference of means over gender, it was observed that there is no significant difference. It is concluded that the ITSON students perceive that they have a capacity for entrepreneurship.
\end{abstract}

Capacity of entrepreneurship, Entrepreneurship, Dimensions of entrepreneurship

Citación: BUELNA-PEÑUÑURI, Ramiro Arnoldo, LÓPEZ-PARRA, María Elvira y ORTEGA-ARREOLA, Jorge. Estudio descriptivo de la capacidad de emprendimiento de los alumnos del Instituto Tecnológico de Sonora. Revista Teoría Educativa. 2019. 3-10: 1-8.

\footnotetext{
* Correspondencia del Autor (correo electrónico: melopez@itson.edu.mx)

$\dagger$ Investigador contribuyendo como primer autor.
} 


\section{Introducción}

La realización de un diagnostico en capacidades de emprendimiento requiere de una metodología, a través de la cual resulta preponderante la consideración de las dimensiones básicas para comprender el problema a resolver mediante una investigación. Por tal motivo, dentro del presente capítulo se presentan los antecedentes del estudio y el problema de estudio. Asimismo, se muestra la relevancia de llevar a cabo está investigación así como su objetivo.

\section{Antecedentes}

Según el Índice Global de Competitividad (IGC) 2018 México ocupa la posición número 46 de 140 economías, publicado en el Foro Económico Mundial (WEF, por sus siglas en inglés) en su Reporte de Competitividad Global 2018-2019.

Desde el sexenio del expresidente Miguel de la Madrid Hurtado se han seguido impulsando políticas públicas que se encargan de regular los asuntos a las actividades financieras del país con el propósito formar un país más competitivo. Sin embargo, los grandes problemas en la economía tales como: baja competitividad, alta desigualdad de los salarios e ingresos, distribución injusta de los recursos públicos, falta de empleo, la existencia de un comercio informal, la falta de oportunidades, alto índice de pobreza y la gran corrupción e impunidad, paraliza el desarrollo económico. Una de las iniciativas para enfrentar la desaceleración económica por parte el presidente de México Andrés Manuel Lopez Obrador fue crear el instituto para devolver al pueblo lo robado el cual destinará lo decomisado al narcotráfico o por actos de corrupción a los municipios con mayor pobreza. Los programas sociales de su gobierno cambiaron las reglas de operación, para que realmente el recurso llegue a sus beneficiados y género iniciativas para el bienestar del pueblo.

El plan nacional de desarrollo (2019) menciona que el objetivo de la política económica no es generar indicadores favorables sino elevar la calidad de vida de los ciudadanos. Los indicadores económicos son un instrumento de medición para observar el panorama de los avances de las variables macroeconómicas que interactúan en la economía mexicana.
El país tiene que avanzar por el camino del crecimiento, austeridad, cero corrupción e impunidad, disciplina fiscal, detener el endeudamiento, respeto a las decisiones del Banco de México, generación de empleos bien remunerados, fortalecimiento del mercado, desarrollar ciencia, educación, investigación y desarrollo tecnológico en los sectores productivos.

En el año 2013 el presidente de los Estados Unidos Mexicanos Lic. Enrique Peña Nieto crea el Instituto Nacional del Emprendedor (INADEM) "es un órgano administrativo desconcentrado de la Secretaría de Economía, que tiene por objeto instrumentar, ejecutar y coordinar la política nacional de apoyo incluyente a emprendedores y a las micro, pequeñas y medianas empresas, impulsando su innovación, competitividad y proyección en los mercados nacional e internacional para aumentar su contribución al desarrollo económico y bienestar social, así como coadyuvar al desarrollo de políticas que fomenten la cultura y productividad empresarial" (INADEM, 2019).

La innovación en Educación Superior es uno de los temas relevantes para la Asociación Nacional de Universidades e Instituciones de Educación Superior (ANUIES) ha impulsado en la necesidad de generar procesos integrales de formación en las diversas áreas temáticas de conocimiento y trabajo de campo profesional. Desde el año 2006 la (ANUIES) propuso transformar los tipos de enseñanza y aprendizaje, innovación, variedad en las modalidades educativas y promover una educación a los individuos para toda vida. La construcción de políticas de innovación en educación superior en un entorno complejo e incierto se ha fomentado. (ANUIES, 2019).

El Instituto Tecnológico de Sonora (ITSON) se ha comprometido incidir en su entorno y en la búsqueda de nuevos proyectos que se vinculen a las áreas académicas dando respuesta a problemas, a partir del 2011 ha desarrollado una Incubadora de empresas dedicada a la prestación de servicios de incubación para empresas de tipo tradicionales, sociales y de base tecnológica para el beneficio de sus egresados para que estos contribuyan al desarrollo económico de la región del sur de Sonora. 
IMSS (2019) reporta que la creación mensual de empleo en enero 2019 es de 94,646 puestos, 46,330 o $95.9 \%$ mayor al promedio de los eneros de los diez años previos $(48,316$ puestos). Este es el segundo mejor aumento reportado en un mes de enero de los últimos diez años. El crecimiento anual en el empleo fue impulsado por el sector de transportes y comunicaciones con $4.8 \%$, agropecuario con $4.6 \%$ y transformación con $4.4 \%$. Instituto Mexicano del Seguro Social. (2019). Es importante para las organizaciones que las universidades fomenten habilidades, conocimientos y actitudes emprendedoras tales como la creatividad, innovación y desarrollo. Penrose (1995) opina que "la capacidad de las empresas para utilizar sus recursos de manera emprendedora les facilita la creación de mecanismos de protección frente a sus competidores y, por lo tanto, la creación de rentas a largo plazo". Portocarrero (2003) llevó a cabo una investigación sobre la capacidad de emprendimiento en estudiantes de Administración y Psicología de la Universidad Nacional Federico Villareal (UNFV) determinando que estudiantes los de Administración presentaban una mayor tendencia a la creación de negocios propios. Obteniendo una alta calificación en Conocimiento de sí mismo, Visión de futuro, Motivación de logro, Planificación, Persuasión. Por otro lado Tinoco (2008) realizo una investigación que determino la capacidad de emprendimiento de los alumnos que ingresaron a la Universidad Nacional Mayor de San Marcos Facultad de Ingeniería Industrial del año 2008 en Perú. Asimismo, se analizaron diferencias en los factores que marcan esta habilidad, como el tipo de formación para ingresar a la universidad, género y colegio de secundaria procedente. Los resultados denotan que los estudiantes cuentan con una capacidad emprendedora alta.

\section{Planteamiento del Problema}

INEGI (2019) menciona que la población ocupada durante el mes de marzo de 2019 por sector de actividad se distribuyó de la siguiente manera: en los servicios se concentró $43.2 \%$ del total, en el comercio $19.5 \%$, en la industria manufacturera $16.7 \%$, en las actividades agropecuarias $11.5 \%$, en la construcción $7.7 \%$, en "otras actividades económicas" (que incluyen la minería, electricidad, agua y suministro de gas) $0.8 \%$ y el restante $0.6 \%$ no especificó su actividad.
El propósito de las Universidades no es solo formar profesionistas competentes, sino individuos capaces de emprender un negocio propio, la falta de empleo es grave y se complica por la situación económica del país, por la educación deficiente que no está enfocada a exigencias y competencias tecnológicas, en un corto tiempo se debe revertir esta situación. De esta forma es de suponer que cuando una persona se prepara a nivel universitario aspira a encontrar un buen trabajo o generar su propia fuente empleo; sin embargo, al momento de egresar existen dificultades para que estos sean autoempleen en su desarrollo laboral, lo que muestra una problemática en la capacidad de emprendimiento.

Por lo anterior surge la siguiente pregunta de investigación: ¿Qué requiere el Instituto Tecnológico de Sonora para conocer las dimensiones de emprendimiento en sus estudiantes?

\section{Justificación}

En caso de llevarse a cabo esta investigación el Instituto Tecnológico de Sonora podría generar beneficios tales como: Impulsar y fortalecer la capacidad de emprendimiento en los estudiantes del sur de Sonora por medio de la materia de emprendimiento la cual la llevan todos los programas educativos que ofrece la institución, la generación de nuevas empresas, nuevos productos y servicios o la innovación de los mismos, la colocación de sus profesionistas emprendedores en el mercado laboral generando empleos directos e indirectos, la mejora y fortalecimiento de sus programas educativos, planes de clase y programas de curso, los egresados de las diferentes carreras buscarán como primera opción el emprendimiento, mayor crecimiento en el desarrollo de las capacidades emprendedoras en sus estudiantes.

En caso de no llevarse a cabo esta investigación el Instituto Tecnológico de Sonora podría tener los siguientes impactos negativos: 
Falta de espíritu emprendedor por parte de los estudiantes ya que la visión será egresar para insertarse en una empresa lo cual no fomenta el autoempleo, desaparición de la incubadora de empresas con la cuenta la institución, falta de contribución al desarrollo económico y social de la región, desaparición de los programas que otorga el gobierno a los emprendedores, la materia de emprendimiento se tendría que eliminar de los programas educativos y aumento en los índices de inseguridad debido a falta de empleo los profesionistas se involucran en actividades ilícitas como narcotráfico, tratante de blancas, secuestros, etc.

\section{Objetivo}

Identificar la capacidad de emprendedora de los estudiantes del Instituto Tecnológico de Sonora que cursan la materia de emprendimiento mediante un diagnostico en cuanto a sus relaciones sociales, realización personal, capacidad creativa y capacidad planificadora para mejorar los programas educativos, planes de clase y programas de curso que la universidad ofrece.

\section{Marco Teórico}

De acuerdo con (Burnett, 2000, citado por Cabana, Plaza, Castillo y Alvarez, 2013) el emprendedor es un individuo que cuenta con liderazgo, planeación, asume de riesgos, evalúa proyectos, y que moviliza recursos desde una zona de bajo rendimiento a una de alta productividad. También puede ser considerado como una persona activa y extrovertida que promueve nuevas ideas e innovaciones y con sus acciones causa desequilibrio en el mercado (Schumpeter, 1942). El emprendedor es aquel empresario creativo e innovador que no cae en la confusión de que cualquier negocio nuevo y pequeño es un emprendimiento, y quien lo lleva a cabo un emprendedor (Formichella, 2004). Emprendedor es una persona que mediante su habilidad creativa y actitud positiva crear una idea innovadora en un negocio, aprovechando las de las oportunidades que se presentan en el entorno y asume los riesgos de fracasar para contribuir al desarrollo de económico de su comunidad. El emprendedor está al pendiente de las oportunidades de negocios que se observan en el entorno la explota y asume los riesgos.
Hay individuos que emprenden un negocio por necesidad, porque tienen que buscar un ingreso extra como una segunda fuente de ingresos para el sustento familiar. Pero hay otros que han realizado estudios universitarios y con esfuerzo aplican lo que han aprendido en el camino de la vida. Pero más allá de las situaciones que te lleven a emprender será el camino que recorras el que te validará como emprendedor.

Mavila, Tinoco \& Campos (2009) definen su modelo y toman en cuenta las siguientes dimensiones para el emprendimiento:

1) Capacidad de realización. Se refiere a retarse a uno mismo y desafiar al futuro, progreso, crecimiento personal, éxito y resultados. Comprende: búsqueda de nuevas oportunidades perseverancia, demanda por eficiencia y eficacia.

2) Capacidad de planificación. Referida a pensar antes de actuar, idenficar metas o resultados que se desean lograr en un tiempo determinado y diseñar planes de acción para mejorar el desempeño aprovechando las oportunidades que se presentan en el entorno y neutralizar las amenazas que ponen en riesgo al negocio.

3) Capacidad de relacionarse socialmente. Aptitud de generar estados de ánimo favorables en los individuos en su entorno que se traduzcan en compromisos de apoyo para el desarrollo ideas innovadoras de negocios. La persona tiene capacidad de establecer vínculos por diferentes razónes con otras personas e organismos que beneficien a su desarrollo. Comprende: persuasión, redes de apoyo y autoconfianza.

4) Capacidad de creatividad. Habilidad para proponer soluciones creativas e innovadoras y propuestas creativas para obtener el éxito en el mundo empresarial. No se trata de copiar sino hacer algo diferente o mejorar algo que ya existe.

\section{Metodología}

El tipo de investigación fue descriptiva, debido a que los datos que se muestran se describen tal y como se obtuvieron de los encuestados. 
Además, es no experimental porque las variables bajo estudio no se manipularon, se analizaron estadísticamente según las respuestas obtenidas en la aplicación del instrumento.

Los sujetos participantes en esta investigación fueron estudiantes de distintas carreras profesionales del Instituto Tecnológico de Sonora (ITSON) que están cursando la materia de emprendimiento en el verano 2019, siendo un total 40 alumnos.

El instrumento que se aplicó fue una adaptación de Mavila, Tinoco y Campos (2009), el cual estuvo integrado en 2 apartados, el primero con 3 preguntas generales del perfil de encuestado y el segundo con un total de 29 Ítems. En esta última parte se incluyeron preguntas de las 4 dimensiones analizadas sobre la capacidad de emprendimiento: (1) planificación, (2) relaciones sociales, (3) relaciones personales y (4) creatividad. Las respuestas se mostraron en una escala de Likert del 5 al 1 donde 5 era Nunca y 1 Siempre.

En cuanto al procedimiento, primero se documentó y analizó el tema relacionado a las capacidades tiene un emprendedor, luego se identificó la población y se determinó que sería una muestra probabilística, pues todos los estudiantes contestarían el instrumento por encontrarse cautivos en las aulas de clase. Posteriormente se analizaron los resultados en el paquete estadístico Statistical Package for the Social Sciences (SPSS) en su versión 21; se hicieron 2 análisis uno relativo a las medias marginales para cada una de las dimensiones antes mencionadas de la capacidad emprendedora y el segundo análisis estuvo relacionado a la diferencia de medias entre las respuestas que observaron las mujeres y los hombres. Se analizó la fiabilidad del instrumento y se obtuvo un Alfa de Cronbach de 0.908. Finalmente se hicieron las discusiones pertinentes y las conclusiones destacadas de la investigación.

\section{Resultados}

En este apartado se muestran tres análisis descriptivos de la capacidad de emprendimiento de los alumnos del ITSON: (1) análisis descriptivo del perfil de los encuestados, (2) análisis de las medias marginales y (3) análisis de diferencia de medias.
Análisis descriptivo del perfil de los encuestados

\begin{tabular}{|l|r|r|}
\hline \multicolumn{1}{|c|}{} & Frecuencia & \multicolumn{1}{c|}{ Porcentaje } \\
\hline Femenino & 21 & 52.5 \\
\hline Masculino & 19 & 47.5 \\
\hline Total & 40 & 100.0 \\
\hline
\end{tabular}

Tabla 1 Genero de los encuestados

Se observa que el $52.5 \%$ de los encuestados son personas del género femenino y el $47.5 \%$, lo que significa que entre los encuestados se tiene un número equilibrado entre hombres y mujeres reportándose 21 personas del género femenino y 19 del género masculino.

\begin{tabular}{|l|r|r|}
\multicolumn{1}{c|}{$\begin{array}{c}\text { Labora } \\
\text { actualmente }\end{array}$} & \multicolumn{1}{c|}{ Frecuencia } & \multicolumn{1}{c|}{ Porcentaje } \\
\hline $\mathrm{Si}$ & 6 & 15.0 \\
\hline No & 34 & 85.0 \\
\hline Total & 40 & 100.0 \\
\hline
\end{tabular}

Tabla 2 Encuestados que laboran

La finalidad de la pregunta sobre la situación laboral de los estudiantes encuestados era identificar si existía una influencia para el emprendimiento, sin embargo como se puede observar el $15 \%$ solamente está trabajando y el $85 \%$ no labora, lo que no se cuenta con elementos suficientes para medir la relación que se tiene sobre la capacidad emprendedora con esta variable.

\section{Análisis de medias marginales}

En las siguientes Tablas 3 y 4 se presentan las medias que tuvieron las respuestas de los estudiantes encuestados con relación a las cuatro dimensiones que integran la capacidad emprendedora como son: (1) capacidad de relaciones sociales, (2) capacidad para las relaciones personales, (3) capacidad para la creatividad y (4) capacidad para la planificación.

\begin{tabular}{|l|r|r|r|r|r|}
\hline $\begin{array}{l}\text { Dimensiones } \\
\text { de la } \\
\text { capacidad } \\
\text { emprendedora }\end{array}$ & Mín. & Máx. & Media & $\begin{array}{c}\text { Desv. } \\
\text { típ. }\end{array}$ \\
\hline $\begin{array}{l}\text { D1 Capacidad } \\
\text { relacionarse } \\
\text { socialmente }\end{array}$ & 40 & 1.17 & 3.17 & 1.995 & .547 \\
\hline $\begin{array}{l}\text { D2 de } \\
\text { Capacidad de } \\
\text { realización } \\
\text { personal }\end{array}$ & 40 & 1 & 3.00 & 1.500 & .494 \\
\hline
\end{tabular}

BUELNA-PEÑUNUURI, Ramiro Arnoldo, LÓPEZ-PARRA, María Elvira y ORTEGA-ARREOLA, Jorge. Estudio descriptivo de la capacidad de emprendimiento de los alumnos del Instituto Tecnológico de Sonora. Revista Teoría Educativa. 2019 


\begin{tabular}{|l|r|r|r|r|r|}
\hline $\begin{array}{l}\text { D3 } \\
\text { Capacidad para } \\
\text { Creatividad }\end{array}$ & 40 & 1 & 3.75 & 1.787 & .616 \\
\hline $\begin{array}{l}\text { D4 } \\
\begin{array}{l}\text { Capacidad de } \\
\text { Planificación }\end{array}\end{array}$ & 40 & 1 & 2.80 & 1.655 & .524 \\
\hline
\end{tabular}

Tabla 3 Medias marginales de las dimensiones

Recordar que las respuestas se mostraron en una escala de Likert del 5 al 1 donde:

5 era Nunca,

4 pocas veces,

3 algunas veces,

2 casi siempre

1 siembre

Por lo tanto, se observa en la Tabla 3, que la dimensión que tiene una media más apegada a la categoría de casi siempre (2), fue la de la capacidad para relacionarse socialmente con una media de 1.995 , lo que quiere decir que los estudiantes casi siempre identifican sus habilidades, proponen nuevas alternativas de solución, colaboran con el equipo de trabajo, actúan por iniciativa propia, construyen redes de apoyo, comprenden las necesidades de sus colaboradores, definen sus metas, influyen en las personas, otorgan soluciones frente a problemas y cumplen con sus compromisos.

Sin embargo, aun cuando la capacidad para relacionarse socialmente fue la mejor calificada por los estudiantes, todas las dimensiones tienen una puntuación que se engloba en la respuesta de casi siempre, con medias de 1.5 para la capacidad de realización personal, media de 1.787 para la capacidad de creatividad y 1.655 para la capacidad de planificación.

En la siguiente Tabla 4 se observan los resultados obtenidos de la media, pero en esta ocasión se colocó en general la capacidad emprendedora que incluye las cuatro dimensiones antes señaladas.

\begin{tabular}{|l|r|r|r|r|r|}
\hline \multicolumn{2}{|c|}{ N } & Mín & Máx & Med. & $\begin{array}{c}\text { Desv. } \\
\text { típ. }\end{array}$ \\
\hline $\begin{array}{l}\text { Capacidad } \\
\text { Emprendedora }\end{array}$ & 40 & 1.15 & 2.81 & 1.802 & .4561 \\
\hline
\end{tabular}

Tabla 4 Media margina de la capacidad emprendedora de los encuestados
En esta Tabla 4 se observa que la media de la capacidad emprendedora fue 1.802 que significa que casi siempre los estudiantes realizan las acciones de relacionarse socialmente con otros, de procurar su realización personal, de considerar su creatividad y de planificar las actividades o acciones para un fin específico.

\section{Análisis de diferencia de medias}

Las hipótesis que se realizaron para hacer la diferencia de medias en cuanto a lo que piensan los Hombres y Mujer sobre su percepción en las capacidades emprendedoras son las siguientes:

H1 (hipótesis alterna): existe una diferencia significativa entre las medias de las percepciones sobre su capacidad emprendedora de mujeres y hombres.

Ho (hipótesis nula): no existe una diferencia, esto es son iguales los resultados

El nivel alfa considerado es de 5\% o .05 . La prueba estadística a utilizar es T de Studen (prueba Paramétrica) para muestras independientes pues es un estudio transversal de 2 grupos (hombres y mujeres) y con una variable aleatoria numérica (capacidad emprendedora).

En cuanto al supuesto de normalidad de la variable capacidad emprendedora, el nivel de significancia de las mujeres fue de 0.117 y el de los hombres fue de 0.217 , como se observa en la Tabla 5 lo que se observa que en ambos casos son mayores que 0.05 (alfa). Por lo tanto los datos se comportan normalmente.

\begin{tabular}{|l|l|r|r|r|}
\hline & Género & \multicolumn{4}{|c|}{ Shapiro-Wilk } \\
& & \multicolumn{4}{c|}{ Estadístico } & gl & \multicolumn{1}{c|}{ Sig. } \\
\hline Capacidad & Femenino & .926 & 21 & .117 \\
\cline { 2 - 5 } Emprendedora & Masculino & .935 & 19 & .217 \\
\hline
\end{tabular}

Tabla 5 Prueba de normalidad

En cuanto al supuesto de igualdad de varianza y de acuerdo a la prueba de Levene, en la Tabla 6 se observa que la significancia fue de .055 y contrastando con el alfa de 0.05 se observa que es mayor que alfa entonces las varianzas de las variables de género son iguales. 


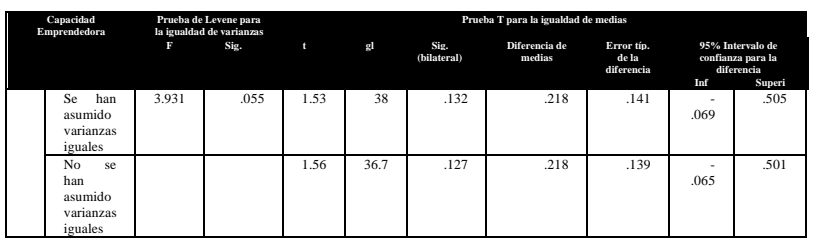

Tabla 6 Prueba de muestras independientes

El valor de la significancia para prueba de $\mathrm{T}$ de Student es de (P-valor) $0.132 \mathrm{y}$ al comparar con 0.05 (alfa), se observa que es mayor que alfa (error) por lo tanto se aceptamos la hipótesis nula, la cual está representada como sigue:

Ho (hipótesis nula): no existe una diferencia significativa de las percepciones de la capacidad emprendedora entre mujeres y los hombres, esto quiere decir que son iguales los resultados según el género.

\section{Conclusiones}

El objetivo de la presente investigación fue identificar la capacidad de emprendimiento de los estudiantes a través de un estudio descriptivo y diferencia de medias, que permita el conocimiento de los niveles de percepción en sus dimensiones, por lo que se concluye que en promedio los estudiantes contestaron que casi siempre con una media de 1.802 realizan las acciones relativas a las capacidades de relacionarse con el entorno, las capacidades de realización personal, la capacidad de planificar y la capacidad para la creatividad.

En cuanto a la percepción que tienen los hombres y las mujeres sobre su capacidad emprendedora, se concluye que no existe diferencia significativa, esto es que sus respuestas fueron relativamente iguales.

El ITSON ha realizado acciones que promueven el desarrollo de capacidades emprendedoras de los estudiantes, como son los cursos presenciales de emprendimiento para todas las carreras, capacitaciones por parte de la coordinación de formación general, además que se cuenta con una incubadora de negocios que tiene las puertas abiertas a las ideas innovadoras de los estudiantes. Al respecto estas actividades se ven reflejadas en la percepción que tienen los alumnos sobre sus capacidades emprendedoras.
Se recomienda que la universidad continúe realizando acciones para el desarrollo emprendedor de los estudiantes, de ahí que esta habilidad empresarial el alumno la pondrá en práctica al crear su propio negocio o bien al formar parte de un organismo en crecimiento y permitirá en lo sucesivo ser más competitiva y asegurar su permanencia en el mercado.

\section{Referencias}

Asociación Nacional de Universidades e Instituciones de Educación Superior. (2019). Acerca del ANUIES. Obtenido de Asociación Nacional de Universidades e Instituciones de Educación Superior: http://www.anuies.mx/programas-yproyectos/proyectos-academicos/innovacionen-educacion-superior

Cabana-Villca, R; Cortes-Castillo, I; PlazaPasten, D; Castillo-Vergara, M \& AlvarezMarin, A. (2013). Análisis de Las Capacidades Emprendedoras Potenciales y Efectivas en Alumnos de Centros de Educación Superior. Journal of Technology Management \& Innovation, 8 (1), 65-75.

FORMICHELLA, L. M. (2004). El concepto emprendimiento y su relación con la educación, el empleo y el desarrollo local. Bueno Aires, Argentina.

Global Entrepreneurship Monitor. (2019). Acerca del GEM. Obtenido de Global Entrepreneurship Monitor: https://gemconsortium.org/economyprofiles/mexico

Instituto Mexicano del Seguro Social. (2019). Acerca del IMSS. Obtenido de Instituto Mexicano del Seguro Social: http://www.imss.gob.mx/prensa/archivo/20190 $2 / 036$

Instituto Nacional del Emprendedor. (2019). Acerca del INADEM. Obtenido de Instituto Nacional del Emprendedor: https://www.inadem.gob.mx/institucional/

Instituto Nacional de Estadística y Geografía. (2019). Acerca del INEGI. Obtenido de Instituto Nacional de Estadística y Geografía: https://www.inegi.org.mx/contenidos/saladepre nsa/boletines/2019/iooe/iooe2019_04.pdf 
Instituto Tecnológico de Sonora. (2019). Acerca del Instituto Tecnológico de Sonora. Obtenido del Instituto Tecnológico de Sonora: https://www.itson.mx/micrositios/incubadora/P aginas/sectores.aspx

Mavila, D; Tinoco, O \& Campos, C. (2009). Factores influyentes en la capacidad emprendedora de los alumnos de la Universidad Nacional Mayor de San Marcos. Revista Facultad de Ingeniería Industrial,12(2) undefined-undefined. [Fecha de consulta 12 de Septiembre de 2019]. pp 32-39 (2009) UNMSM ISSN: 1560-9146 (Impreso)/ ISSN: 1810-9993 (Electrónico). Disponible en: https://revistasinvestigacion.unmsm.edu.pe

Krauss, C. (2005). Las actitudes Emprendedoras en los Estudiantes de la Universidad Católica del Uruguay. Revista FCE , 3-4

Penrose, E. (1995): "Research on the business firm limits to the growth and size of firms", The American Economic Review,45 (2), pp. 531543.

Plan nacional de desarrollo. (2019). Acerca del plan nacional de desarrollo. Obtenido de: Plan nacional de https://lopezobrador.org.mx/wpcontent/uploads/2019/05/PLAN-NACIONALDE-DESARROLLO-2019-2024.pdf

Portocarrero, C. (2003). Capacidad emprendedora y calificación empresarial en estudiantes de Administración y Psicología de la UNFV. Tesis de Maestría UNFV. Lima.

Schumpeter. J. A. (1942). Capitalismo, socialismo y democracia. Estados Unidos: Gerorge Allen y Unwin

Secretaria de Gobernación. (2019). Acerca del Plan nacional de desarrollo 2019-2024 obtenido de https://www.gob.mx/cenace/acciones-yprogramas/plan-nacional-de-desarrollo-20192024-195029

Tinoco, O. (2008). Medición de la capacidad emprendedora de ingresantes a la facultad de Ingeniera Industrial de la UNMSM. Industria Data, 11(2), undefined-undefined. [Fecha de consulta 12 de Septiembre de 2019]. ISSN: 1560-9146. Disponible en: https://www.redalyc.org/pdf/816/81619829003. pdf
Reporte Globlal de Competitividad. Acerca del Reporte Globlal de Competitividad obtenido de Reporte Globlal de Competitividad: https://www2.deloitte.com/content/dam/Deloitt e/cr/Documents/finance/docs/181017-cr-fasReporte-Global-de-Competitividad-WEF.pdf 